\title{
Estudo de avaliação das habilidades de vida independente entre usuários da rede de atenção psicossocial do Rio Grande do Sul
}

\section{Evaluation study of independent living skills among users of psychosocial care network in Rio Grande do Sul}

\author{
Candida Garcia Sinott Silveira Rodrigues ${ }^{1}$, Vanda Maria da Rosa Jardim¹, \\ Luciane Prado Kantorski ${ }^{1}$, Valéria Christina Cristello Coimbra ${ }^{1}$, \\ Carlos Alberto dos Santos Treichel ${ }^{1}$, Beatriz Franchini ${ }^{1}$, \\ Andréia Simone Ferreira Bretanha ${ }^{1}$, Aline dos Santos Neutzling ${ }^{1}$
}

\begin{abstract}
Resumo
Introdução: Este estudo objetivou descrever as habilidades de vida independente de uma amostra de usuários da rede de atenção psicossocial do Rio Grande do Sul, identificando as áreas com menores e maiores desempenhos. Métodos: A amostra foi de 390 usuários. A fim de determinar o grau de habilidades da vida cotidiana de pacientes psiquiátricos, foi utilizada a escala Independent Living Skills Survey (ILSS), que avalia o desempenho dos sujeitos em nove áreas de atividade da vida cotidiana. As análises foram conduzidas no pacote estatístico Stata 11, e utilizou-se a estatística descritiva para apresentação dos resultados. Resultados: O escore global encontrado para habilidades de vida independente foi de 2,44, com desvio-padrão (DP) de 0,90. Os itens de maior escore foram aqueles relacionados à alimentação, cuidados pessoais e transporte. Os itens com menores escores foram emprego, lazer e administração de dinheiro. Conclusão: As habilidades de vida independente entre os indivíduos incluídos neste estudo foram maiores do que em outras pesquisas realizadas anteriormente acerca do tema.
\end{abstract}

Palavras-chave: autonomia pessoal; vida independente; saúde mental; serviços comunitários de saúde mental; ILSS-BR.

\begin{abstract}
Introduction: This study aims to describe the independent life skills from a sample of psychosocial care network users in the state of Rio Grande do Sul indicating the areas of higher and lower performances. Methodology: The sample comprises 390 users. For rating the amount of everyday life skills from the psychiatric patients, we applied the Independent Living Skills Survey (ILSS) scale, which evaluates the users regarding 9 everyday day life activities. The analyses were run by the statistic package Stata 11 and were presented as descriptive statistics. Results: The global score for independent living skills was 2.44 with a standard deviation $\mathrm{SD}=0.90$. The fields with highest score were those related to feeding, personal cares and transportation. Those with lower scores were related to job, recreation and money management. Conclusion: The independent living skills among the individuals included in this study were higher than among previous studies on the subject.
\end{abstract}

Keywords: personal autonomy; independent life; mental health; community mental health services; ILSS-BR.

${ }^{1}$ Faculdade de Enfermagem, Universidade Federal de Pelotas (UFPel) - Pelotas (RS), Brasil.

Trabalho realizado em Serviços Residenciais Terapêuticos e Centros de Atenção Psicossocial dos municípios de Alegrete (RS), Bagé (RS), Caxias do Sul (RS), Viamão (RS) e Porto Alegre (RS), Brasil.

Endereço para correspondência: Candida Garcia Sinott Silveira Rodrigues - Rua Gomes Carneiro, 1 - Porto - CEP: 96010-110 - Pelotas (RS), Brasil -

Email: candidasinott@hotmail.com

Fonte de financiamento: MCT-CNPq/CT- Saúde/ MS-SCTIE-DECIT / 33/2008.

Conflito de interesses: nada a declarar. 


\section{INTRODUÇÃO}

Por muito tempo a psiquiatria focou seu olhar na doença, deixando o ser humano de lado, o que ocasionou uma objetificação do sujeito. Muito se preocupou em definir a doença, classificá-la e gerenciá-la, codificar normas e sintomas, já que a ciência afirmava que a pessoa com transtorno mental era produto de alteração essencialmente biológica e que nada se poderia fazer a não ser aceitar sua condição de anormalidade ${ }^{1}$.

Essa prática, centrada na definição da loucura, limitava o sujeito à sua condição psíquica e deixava de considerar sua subjetividade, de forma que o indivíduo terminava por ter sua cidadania e identidade roubadas, além de ser privado da liberdade por meio da lógica asilar manicomial ${ }^{1}$.

As críticas e a oposição à institucionalização e aos manicômios surgiram a partir da realidade de maus-tratos, da violência e negação da cidadania e dignidade no cuidado e no tratamento às pessoas com transtorno mental, uma vez que não era aceitável que indivíduos continuassem a ser expostos a situações desumanas em função de sua condição psíquica² .

Entre os principais movimentos críticos à tradição psiquiátrica no mundo, ao longo do século XX, destacam-se os movimentos inglês, francês e norte-americano. Contudo, foi na década de 1970 que ocorreu na Itália uma série de experiências de psiquiatria denominada democrática, que acabou por influenciar o movimento de reforma psiquiátrica no Brasil ${ }^{2}$.

Inspirada no modelo italiano, essa reforma propunha não uma reformulação ou melhora no manicômio, mas uma ruptura com o cuidado focado na doença e na relação de poder entre o saber médico e a loucura ${ }^{1,2}$.

Nesse contexto, atualmente, a Política Nacional de Saúde Mental brasileira tem a desinstitucionalização como seu eixo organizador. Trata-se de um processo que, além da desospitalização de moradores de hospitais psiquiátricos, visa à construção de condições efetivas para um cuidado comunitário contínuo e qualificado para todos os que necessitam de tratamento, reabilitação e reinserção social ${ }^{3}$.

Os movimentos de desinstitucionalização ocorridos no país por meio da reforma psiquiátrica levaram ao estabelecimento de um modelo de atenção psicossocial de base comunitária, orientado, principalmente, por serviços como os Centros de Atenção Psicossocial (CAPS) e os Serviços Residenciais Terapêuticos (SRT). Esses serviços visam acompanhar os usuários na comunidade, oferecer estruturas de suporte social e promover a reabilitação plena, já que os usuários - indivíduos em sofrimento psíquico - estão susceptíveis a dificuldades na realização das atividades cotidianas, na construção de seus laços afetivos/sociais e no desenvolvimento de sua autonomia.

Com o estabelecimento desses serviços, o desafio tem sido a efetividade das ações em saúde mental, na construção da reabilitação psicossocial dos usuários e na desmistificação da loucura na sociedade. Para isso, a Organização Mundial de Saúde tem proposto, desde 2001, avaliação dos serviços e programas de saúde mental, principalmente no que diz respeito à satisfação com o serviço, qualidade de vida, grau de autonomia, comportamento social e impacto dos programas de reabilitação psicossocial na vida dos sujeitos com sofrimento psíquico ${ }^{4}$.

Especialmente sobre a avaliação da autonomia, são evidenciados na literatura investimentos importantes que situam esse tipo de avaliação como um marcador de efetividade dos serviços de saúde mental de base comunitária ${ }^{5-7}$. Contudo, a necessidade de avanços nesse processo avaliativo é continuamente tensionada pelos órgãos internacionais. O plano de ação em saúde mental da Organização Mundial da Saúde para o período de 2013 a 2020, por exemplo, indica a demanda de formação de uma base de evidencias quanto à avaliação da autonomia a fim de identificar a efetividade dos serviços e estabelecer programas comunitários cada vez mais responsivos 8 .

Esses tensionamentos surgem à medida que a identificação do grau de autonomia nas diversas áreas da vida cotidiana dos indivíduos atendidos pelos serviços consiste em uma estratégia potente a fim de determinar os objetivos mais pertinentes para os programas de reabilitação, além de possibilitar que se monitorem, posteriormente, os efeitos desses programas na vida dos sujeitos ${ }^{5}$.

No Brasil, os tensionamentos quanto a essa perspectiva levaram à validação de uma escala que fosse capaz de identificar as habilidades de vida independente dos usuários de serviços de saúde mental. Trata-se do instrumento Independent Living Skills Survey (ILSS), elaborado por Wallace 9

Embora seja considerado um instrumento potente de avaliação, sua aplicação ainda é escassa, havendo poucos registros na literatura quanto à sua utilização. Portanto, no sentido de contribuir para avaliação das práticas reabilitadoras no cotidiano dos serviços de saúde mental, por meio da construção de uma base de evidências quanto aos resultados dos serviços na vida dos sujeitos, este estudo teve por objetivo descrever as habilidades de vida independente dos usuários da rede de atenção psicossocial do Rio Grande do Sul.

\section{METODOLOGIA}

Trata-se de um estudo transversal, recorte do projeto "Redes que reabilitam - avaliando experiências inovadoras de composição de redes de atenção psicossocial (REDESUL)", realizado em 2009 e financiado pelo MCT-CNPq/CT-Saúde/ MS-SCTIE-DECIT n ${ }^{\circ} 33 / 2008$.

Foram selecionados para o estudo todos os municípios do Rio Grande do Sul que contavam com SRT em sua rede durante a execução da pesquisa: Alegrete, Bagé, Caxias do Sul, Viamão e Porto Alegre. 
Para o delineamento da amostra, o cálculo foi efetuado no software Epi Info 6.04, considerando um valor de alfa igual a 5\% e poder de $80 \%$. Diferentes medidas de variabilidade encontradas na literatura foram consideradas, buscando obter valores com menor possibilidade de erro. Considerando os subgrupos e as possíveis perdas, foram acrescidos $20 \%$ ao maior valor de amostra calculado, demandando um $\mathrm{N}=450$. $\mathrm{O}$ total de perdas, relacionadas a recusas e dificuldade em acessar os usuários do serviço, foi de 60 indivíduos, ou seja, 13,3\% da projeção inicial, resultando em 390 usuários.

A seleção dos entrevistados se deu em dois momentos. No primeiro foram acessados intencionalmente todos os usuários dos SRT inseridos no estudo, totalizando 120 indivíduos. Posteriormente, foram identificados, por meio dos registros dos CAPS, os sujeitos que utilizavam o serviço na modalidade intensivo e semi-intensivo, selecionando-os proporcionalmente nas duas modalidades de forma aleatória. Dessa forma, foram incluídos na amostra 134 usuários de CAPS da modalidade intensivo e 134 da modalidade semi-intensivo (20 utilizavam concomitante CAPS e SRT).

Na perspectiva de avaliar o grau de habilidades da vida cotidiana de pacientes psiquiátricos, foi utilizada a escala Inventário de Habilidades de Vida Independente (ILSS-BR), adaptada e validada para o Brasil por Lima et al. ${ }^{5}$ e por Bandeira et al. ${ }^{10} \mathrm{a}$ partir da escala original ILSS, elaborada por Wallace em 1986.

O referido instrumento foi desenvolvido no intuito de avaliar a autonomia de pacientes psiquiátricos em nove áreas da vida cotidiana, em termos da frequência com que eles apresentavam as habilidades básicas para funcionar de forma independente na comunidade.

A versão do ILSS-BR para o contexto brasileiro resultou, após o estudo de tradução e de adaptação transcultural e as análises estatísticas realizadas, em uma escala de 84 itens, dividida em nove subescalas, uma para cada área da vida cotidiana. Dessa forma, o instrumento inclui: 8 itens referentes à alimentação; 13 , aos cuidados pessoais; 12 , às atividades domésticas; 7 , ao preparo e armazenamento de alimentos; 8 , à saúde; 12 , à administração do dinheiro; 7 , ao transporte; 9 , ao lazer; 8 itens, à procura e/ou conservação do emprego.

O instrumento avalia, em uma escala do tipo Likert de 5 pontos, a frequência com que o paciente realizou, no último mês, as atividades cotidianas necessárias ao seu funcionamento independente na comunidade ( $0=$ nunca; $1=$ algumas vezes; $2=$ com frequência; $3=$ na maioria das vezes; $4=$ sempre). Para se avaliar o nível de funcionamento dos pacientes em relação às nove áreas de habilidades da vida cotidiana independente, calcula-se o escore médio das respostas obtidas em cada uma das nove subescalas do ILSS-BR. Essa média, que pode variar de 0 a 4 , indica um nível mais elevado de habilidades de vida independente quanto mais próxima estiver do valor máximo 4 . No mesmo sentido, o escore global se dá por meio do escore médio de todas as subescalas.
A construção e o processamento dos dados deram-se por meio do software Epi Info, com dupla digitação e limpeza do banco de dados por digitadores independentes. O controle de qualidade contou com a replicação de $10 \%$ das entrevistas realizadas. As análises foram conduzidas com o pacote estatístico Stata 11 (Stata Corp., College Station, Estados Unidos). Para apresentação dos resultados, foi utilizada a estatística descritiva.

O estudo foi aprovado pelo Comitê de Ética em Pesquisa da Faculdade de Odontologia da Universidade Federal de Pelotas, sob Parecer $n^{\circ}$ 073/2009. A pesquisa também encontra-se em concordância com os procedimentos éticos exigidos para pesquisa com seres humanos, atendendo ao constante da Resolução no 196/96 do Conselho Nacional de Saúde e também a resolução vigente CNS nº 466/2012.

\section{RESULTADOS}

A amostra deste estudo foi de 390 sujeitos, dos quais 120 eram usuários de SRT, 134, de CAPS na modalidade intensiva, e 134, de CAPS na modalidade semi-intensiva (20 sujeitos usavam concomitantemente CAPS e SRT). Sua composição compreendeu majoritariamente indivíduos do sexo masculino (52,6\%), com 40 anos ou mais $(62,4 \%)$, autodeclarados brancos $(55,2 \%)$ e solteiros $(78,6 \%)$.

Os resultados referentes às medidas da escala ILSS-BR estão dispostos na Tabela 1, na qual é possível observar as médias e o desvio-padrão dos escores apresentados em cada subescala proposta pelo instrumento, além do escore e desvio-padrão global.

Por meio da análise dos dados dispostos na Tabela 1, é possível observar que o escore global entre a população estudada foi de $2,44(\mathrm{DP}=0,90)$. As áreas com maiores escores foram aquelas relativas à alimentação $(3,29 ; \mathrm{DP}=0,71)$, cuidados pessoais $(3,08 ; \mathrm{DP}=0,92)$ e transporte $(2,74 ; \mathrm{DP}=1,15)$. No entanto, as áreas avaliadas com menores escores foram aquelas referentes à emprego $(1,0 ; \mathrm{DP}=1,19)$, lazer $(1,94 ; \mathrm{DP}=1,03)$ e administração de dinheiro $(2,06 ; \mathrm{DP}=1,44)$.

Tabela 1. Média e desvio-padrão dos escores global e de cada subescala avaliada pela ILSS-BR entre usuários da rede de atenção psicossocial do Rio Grande do Sul

\begin{tabular}{lcc} 
Subescala & Média & $\begin{array}{c}\text { Desvio- } \\
\text { padrão }\end{array}$ \\
Alimentação & 3,29 & 0,71 \\
Cuidados pessoais & 3,08 & 0,92 \\
Atividades domésticas & 2,50 & 1,37 \\
Preparo e armazenamento de alimentos & 2,55 & 1,39 \\
Saúde & 2,68 & 0,92 \\
Administração de dinheiro & 2,06 & 1,41 \\
Transporte & 2,74 & 1,15 \\
Lazer & 1,94 & 1,03 \\
Emprego & 1,0 & 1,19 \\
Escore global & 2,44 & 0,90 \\
\hline
\end{tabular}


Quanto às demais áreas, evidenciou-se um escore de 2,50 $(\mathrm{DP}=1,37)$ para atividades domésticas, de $2,55(\mathrm{DP}=1,39)$ para preparo e armazenamento de alimentos e de $2,68(\mathrm{DP}=0,92)$ para saúde.

\section{DISCUSSÃO}

O escore global encontrado para habilidades de vida independente nesta pesquisa por meio da escala ILSS-BR foi de 2,44, com desvio-padrão de 0,90. Embora haja escassez de estudos prévios quanto às habilidades para vida independente de indivíduos em sofrimento psíquico no contexto brasileiro, limitando possíveis generalizações, a comparação desse resultado com aqueles obtidos por estudos anteriores sugere um cenário positivo quanto à avaliação das habilidades de vida independente dos sujeitos estudados.

As pesquisas realizadas até então indicam escores menores que os observados pelo presente trabalho. Na validação dessa escala realizada por Lima et al. ${ }^{5}$, por exemplo, foi encontrado um escore global de 1,51 . No mesmo sentido, Bandeira et al. ${ }^{10}$, ao realizarem um estudo de teste quanto às qualidades psicométricas da escala, obtiveram um escore global de 1,68. Já Vidal et al. ${ }^{7}$ evidenciaram, na primeira fase de seu estudo, um escore global de 1,70, com desvio-padrão de 0,62. Já Wagner et al. ${ }^{6}$ encontraram escores ainda menores: escore global de 1,13 , com desvio-padrão de 0,79 .

Com base nesses dados, de forma geral pode-se dizer que os indivíduos incluídos neste estudo apresentaram maiores habilidades de vida independente. No entanto, cabe ressaltar as diferenças entre as amostras desta e das pesquisas citadas anteriormente. Tanto Bandeira et al. ${ }^{10}$ como Wagner et al. ${ }^{6}$ realizaram seus estudos em instituições psiquiátricas voltadas para indivíduos que, após longos históricos de internação, perderam seus vínculos familiares.

Como comparação, cabe destacar o trabalho de Vidal et al. ${ }^{7}$, realizado em dois momentos. Na primeira fase, quando as pessoas estavam vinculadas a um hospital psiquiátrico, os autores evidenciaram um escore global de $1,70(\mathrm{DP}=0,62)$. Já na segunda fase, após dois anos da transferência desses indivíduos para residenciais terapêuticos, o escore encontrado foi de 2,29 ( $\mathrm{DP}=0,87)$.

Embora a amostra deste trabalho não seja composta unicamente por indivíduos oriundos de SRT, pode-se dizer que ela é semelhante aos indivíduos estudados por Vidal et al. ${ }^{7}$ na segunda fase de seu estudo. É possível apontar ainda que os escores encontrados por ambos os estudos foram mais próximos se comparados aos demais citados anteriormente.

Estudos com recortes longitudinais parecem ser uma ótima fonte de dados quanto ao comportamento das habilidades de vida independente dos sujeitos à medida que estes são inseridos em diferentes perspectivas de cuidado. Dias ${ }^{11}$, ao avaliar um grupo de usuários de SRT em momentos distintos com intervalo de dois anos, encontrou melhores resultados entre os usuários em sete das nove subescalas da ILSS-BR.

Portanto, levando em conta que o SRT é um serviço focado na reabilitação psicossocial do sujeito, os resultados obtidos por Vidal et al. ${ }^{7}$ e Dias ${ }^{11}$, somados aos resultados deste estudo, quando comparados àqueles desenvolvidos em instituições de cunho hospitalocêntrico, sugerem-nos que projetos em conformidade com o proposto pela atual política de saúde mental no Brasil consistem em mecanismos potentes de transformação na vida dos usuários no que diz respeito à sua autonomia.

É possível observar que das noves subescalas avaliadas, seis apresentaram escores iguais ou superiores a 2,5 (metade da maior pontuação possível), indicando, assim, um desempenho no mínimo razoável para estas áreas (alimentação; cuidados pessoais; atividades domésticas; preparo e armazenamento de alimentos; saúde; transporte). As áreas com maiores escores foram aquelas relativas à alimentação $(3,29 ; \mathrm{DP}=0,71)$, cuidados pessoais $(3,08 ; \mathrm{DP}=0,92)$ e transporte $(2,74 ; \mathrm{DP}=1,15)$.

Dessa forma, pode-se apontar que os resultados deste estudo assumem direção semelhante aos da pesquisa de Sprandel et al. ${ }^{12}$, que investigaram as contribuições do CAPS para o desenvolvimento da autonomia dos usuários. Os resultando de ambos os estudos convergem para evidência da contribuição do serviço, em especial para o resgate das habilidades para as atividades cotidianas.

Essa é uma perspectiva positiva à medida que vai ao encontro dos tensionamentos da política atual de saúde mental brasileira, balizada pela perspectiva de reabilitação psicossocial. Ressalta-se que esse é um processo que busca romper com o paradigma de clínica baseada na díade doença-cura, evoluindo para uma relação existência-cuidado, a qual, para além da remissão de sintomas, visa à potencialização das capacidades do indivíduo para o estabelecimento da vida autônoma ${ }^{13}$.

Contudo, se, por um lado, a abordagem aqui proposta quanto às habilidades de vida independente dos usuários de serviços comunitários permite identificar áreas da vida do sujeito que têm sido beneficiadas pela abordagem de reabilitação psicossocial, por outro permite identificar aquelas que ainda necessitam de práticas inovadoras, mas que auxiliem no resgate da autonomia e efetivem transformações de vida, sempre respeitando a singularidade dos sujeitos.

Nesse sentido, os resultados deste estudo apontam que as áreas avaliadas, para as quais foram encontrados os escores menores, dizem respeito à administração de dinheiro $(2,06$; $\mathrm{DP}=1,41)$, lazer $(1,94 ; \mathrm{DP}=1,03)$ e emprego $(1,0 ; \mathrm{DP}=1,19)$. Tendo em vista que os escores encontrados para essas áreas foram todos inferiores a 2,5 (metade da maior pontuação possível), sugere-se um baixo desempenho dos indivíduos estudados em relação a esses aspectos.

Ressalta-se que os programas de reabilitação psicossocial devem estar atentos às necessidades dos usuários nos diversos aspectos da vida dos sujeitos. Saraceno ${ }^{13}$, um importante representante 
das propostas de reabilitação psicossocial, defende que esse processo só pode se dar por meio da plena contratualidade em três grandes cenários: hábitat, rede social e trabalho com valor social. Logo, é necessário que se estabeleça um olhar atento para que as medidas terapêuticas propostas pelos serviços sejam capazes de estimular, além da instrumentalização para as atividades cotidianas, a capacidade do sujeito de estabelecer condições plenas de vida autônoma, por meio da criação de vínculos sociais e estímulo à inserção no mercado de trabalho.

É preciso salientar que não há, no instrumento utilizado por este estudo, itens que possam dar subsídio para uma medida quanto ao estabelecimento das relações sociais dos indivíduos estudados, o que evidencia uma limitação da pesquisa diante das perspectivas da reabilitação psicossocial.

Quanto às áreas avaliadas com baixos escores, pode-se apontar que o fraco desempenho quanto à subescala referente ao lazer vai contra resultados prévios observados na literatura. Embora estudos como o de Sprandel et al. ${ }^{12}$ indiquem que uma das principais contribuições dos serviços comunitários de saúde mental identificadas por usuários seja relativa a maiores oportunidades de atividades de lazer, o escore para essa área foi de 1,94, com desvio-padrão de 1,03.

No entanto, há de se levar em conta os itens avaliados pelo instrumento nessa área. Entre as nove questões da subescala lazer, é possível identificar alguns recursos que caíram em desuso ao longo dos anos. O envio de cartas como forma de comunicação, por exemplo, é muito pouco utilizado na sociedade atual, bem como os jogos de mesa. Outra questão que merece destaque nesse sentido é quanto à manutenção de jardins, os quais, devido à urbanização e à expansão de moradias do tipo apartamento, são cada vez menos presentes na vida das pessoas.

Nesse sentido, como a elaboração da escala advém de 1985, talvez devam ser considerados alguns ajustes quanto às questões investigadas a fim de aproximá-las do modo de vida atual.

Quanto às áreas administração de dinheiro e emprego, existem características dos sujeitos estudados que precisam ser levadas em conta para interpretação dos resultados. A amostra deste estudo compreendeu majoritariamente usuários de CAPS, ou seja, indivíduos que vivem com suas famílias, as quais, em grande parte dos casos, podem ser chefiadas por outras pessoas que não o usuário. Logo, a administração do dinheiro pode ser permeada pelo modus operandi da família, de forma que o chefe familiar seja a pessoa responsável por gerenciar os gastos. Sendo assim, muitos usuários não teriam a oportunidade de vivenciar essa experiência; portanto, o escore referente a diversas questões relacionadas a esse item poderia diminuir.

Outro fator que deve ser considerado é que 5,6\% dos sujeitos possuem emprego formal, outros $13,7 \%$ dependem da renda familiar, mas a grande maioria é aposentada/pensionista ou recebe auxílios (29,8 e 50,9\% respectivamente), ou seja, estariam sujeitos aos eventos dispostos no item referente a emprego apenas aqueles cuja fonte de renda advém de trabalho formal ou renda familiar.
Ressalta-se que estudos anteriores já evidenciaram dificuldades de acesso a essas áreas. No estudo de Vidal et al. ${ }^{7}$, por exemplo, as subescalas referentes à administração de dinheiro e emprego foram suprimidas devido à inaplicabilidade dos itens incluídos na avaliação ao perfil da população estudada.

Contudo, os investimentos realizados por este e pelos demais estudos que utilizaram o instrumento até então constituem um movimento importante no sentido de contribuir para a avaliação dos resultados de serviços comunitários de saúde mental na vida dos usuários. Ressalta-se que a construção de uma base de evidências quanto aos resultados, em especial sobre a autonomia dos sujeitos, é o primeiro passo para a avaliação da efetividade dos serviços ${ }^{8}$. A fim de avançar, um investimento importante talvez seja a identificação dos fatores individuais que contribuem para melhores ou piores desempenhos, já que as análises conduzidas até então são realizadas por agrupamentos.

Uma limitação desta pesquisa é a data de coleta das informações. No entanto, devido à escassez de trabalhos na área e à potencialidade em se tornar uma ferramenta importante na proposição de trabalhos futuros, os achados continuam relevantes no contexto atual.

\section{CONCLUSÃO}

As habilidades de vida independente entre os indivíduos incluídos neste estudo foram maiores do que em outras pesquisas realizadas anteriormente acerca do tema. Os itens de maior escore foram aqueles relacionados à alimentação, cuidados pessoais e transporte. Os itens com menores escores foram emprego, lazer e administração de dinheiro.

Nesse sentido, se, por um lado, a abordagem quanto às habilidades de vida independente dos usuários de serviços comunitários permite identificar áreas da vida do sujeito que têm sido beneficiadas pela abordagem de reabilitação psicossocial, por outro permite identificar aquelas que ainda necessitam de práticas inovadoras, mas que auxiliem no resgate da autonomia e efetivem transformações de vida, sempre respeitando a singularidade dos sujeitos.

Dessa forma, este estudo tenciona a necessidade de práticas inovadoras, em especial no estímulo das potencialidades dos indivíduos atendidos por serviços comunitários de saúde mental em relação à administração de dinheiro e emprego, aspectos que vão ao encontro de um dos pilares básicos das propostas de reabilitação psicossocial, o trabalho com valor social.

Para futuras pesquisas na área, sugere-se considerar alguns ajustes quanto às questões investigadas na abordagem das habilidades para vida independente a fim de aproximá-las ao modo de vida atual. Sugere-se ainda a inclusão de uma medida quanto ao estabelecimento das relações sociais dos indivíduos estudados, já que ela pode dar subsídio a uma avaliação mais completa de todas as interfaces das propostas de reabilitação psicossocial. 


\section{REFERÊNCIAS}

1. Yasui S. Rupturas e encontros: desafios da Reforma Psiquiátrica Brasileira. Rio de Janeiro: Fiocruz; 2010.

2. Basaglia F. A instituição negada: relato de um hospital psiquiátrico. Rio de Janeiro: Graal; 1985.

3. Brasil. Ministério da Saúde. Secretaria de Atenção à Saúde. Departamento de Ações Programáticas Estratégicas. Coordenação Geral de Saúde Mental, Álcool e Outras Drogas. Saúde Mental no SUS: cuidado em Liberdade, Defesa de Direitos e Rede de Atenção Psicossocial. Relatório de Gestão 2011-2015. Brasília; 2016.

4. World Health Organization. Atlas de salud mental em el mundo 2001: determinantes de la salud mental y poblaciones. Genebra: Departamento de Salud Mental y Toxicomanías; 2001.

5. Lima LA, Bandeira M, Gonçalves S. Validação transcultural do Inventário de Habilidades de Vida Independente (ILSS-BR) para pacientes psiquiátricos. J Bras Psiquiatr. 2003;52:143-58.

6. Wagner LC, Fleck MPA, Wagner M, Dias MTG. Autonomy of long-stay psychiatric inpatients. Rev. Saúde Públ. 2006;40(4):699-705.

7. Vidal CEL, Gontijo ECDM, Bandeira MB. Avaliação das habilidades de vida independente e comportamento social de pacientes psiquiátricos desospitalizados. Rev Psiquiatr Rio Gd Sul. 2007;29(3):294-304.
8. World Health Organization. Comprehensive mental health action plan 2013-2020. Genebra; 2013

9. Wallace CJ. Functional assessment in rehabilitation. Schizophr Bull. 1986;12:604-30.

10. Bandeira M, Lima LA, Gonçalves S. Qualidades psicométricas no papel da Escala de Habilidades de Vida Independente de pacientes psiquiátricos (ILSSBR): fidedignidade do teste e do reteste. Rev. Psiq. Clín. 2003;30(4):121-5.

11. Dias MTG. A reforma psiquiátrica brasileira e os direitos dos portadores de transtorno mental: uma análise a partir do serviço residencial terapêutico morada São Pedro [tese]. Porto Alegre: Faculdade de Serviço Social; 2007.

12. Sprandel LIS, Jardim VMR, Kantorski LP. A contribuição do Centro de Atenção Psicossocial no processo de autonomia dos usuários. Ciência, Cuidado e Saúde. 2008;7(2):1-4.

13. Saraceno B. Reabilitação psicossocial: uma estratégia para a passagem do milênio. In: Pitta AM. Reabilitação psicossocial no Brasil. São Paulo: Hucitec; 1996.

Recebido em: Abr. 03, 2016 Aprovado em: Set. 15, 2016 